\title{
A Two-Stage Algorithm of Locational Marginal Price Calculation Subject to Carbon Emission Allowance
}

\author{
Mingxing $\mathrm{Wu}^{1}{ }^{1}$, Zhilin $\mathrm{Lu}^{2, *}$, Qing Chen ${ }^{1}$, Tao $\mathrm{Zhu}^{1}{ }^{1}$, En Lu ${ }^{1}$, Wentian $\mathrm{Lu}^{2}$ and Mingbo Liu ${ }^{2} \mathbb{D}$ \\ 1 Guangdong Power Exchange Center Co., Guangzhou 510080, China; wumingxing@gd.csg.cn (M.W.); \\ chenqing@gd.csg.cn (Q.C.); zhutao@gd.csg.cn (T.Z.); luen@gd.csg.cn (E.L.) \\ 2 School of Electric Power Engineering, South China University of Technology, Guangzhou 510640, China; \\ hnlgtiantian@163.com (W.L.); epmbliu@scut.edu.cn (M.L.) \\ * Correspondence: epzhll@163.com; Tel.: +86-156-2278-3757
}

Received: 9 March 2020; Accepted: 14 May 2020; Published: 15 May 2020

\begin{abstract}
To analyze the effect of carbon emission quota allocation on the locational marginal price (LMP) of day-ahead electricity markets, this paper proposes a two-stage algorithm. For the first stage of the algorithm, a multi-objective optimization model is established to simultaneously minimize the total costs and carbon emission costs of power systems. Hence, an evenly distributed Pareto optimal solution can be solved effectively by means of the normalized normal constraint method. For the second stage, a tracing model is built with the goal of minimizing the total costs of power systems and satisfying the constraints generated based on the Pareto optimal solution obtained from the first stage. Furthermore, the influence of carbon emission quota allocation on the LMP of electricity markets is analyzed, and different schemes to allocate carbon emission quotas are evaluated on a real 1560-bus and 52-unit system.
\end{abstract}

Keywords: carbon emission allowance; day-ahead electricity market; multi-objective optimization; two-stage algorithm

\section{Introduction}

With the rapid development of the modern industrial system and the overconsumption of resources and energy, huge amounts of greenhouse gases have been emitted into the atmosphere over the last 20 years, which has brought about environmental pollution and damage to the ecosystem [1-3]. Therefore, numerous industrialized countries have promoted international actions to reduce carbon emissions and achieve sustainable development $[4,5]$. In the European Union, many countries have committed to reducing carbon emissions by $20 \%$ by 2025 , and $45 \%$ by 2050 , in order to limit global temperature rise [6]. As a major contributor of carbon emissions, China set the goal of reducing carbon emissions by $40 \%-50 \%$ by 2020 compared to 2005 [7,8]. Within this context, in addition to vigorously developing the renewable energy industries, many countries also utilize financial incentives such as carbon emission trading (CET) to encourage emission reduction. China has already started CET pilots in seven provinces or cities, including Guangdong Province, and planned to further promote CET mechanisms across the country. Moreover, the government executed the pilot operation of provincial spot electricity markets in Guangdong, Mengxi, Zhejiang, Shanxi, Shandong, Fujian, Sichuan, and Gansu at the end of 2018.

According to studies on greenhouse gas emissions, the carbon emissions of the power generation industry by fossil fuel combustion account for a very high proportion of the total emissions in many countries, especially in developing countries. In China, over $40 \%$ of carbon emissions are produced by fossil fuel combustion during the process of power generation [9]. Therefore, the implementation of the CET mechanism has a significant impact on the development of the power industry, and it is 
significant to study how the CET mechanism and the carbon emission quota allocation mechanism will impact the electricity market in China at this stage, especially the marginal price of the spot market.

To date, numerous valuable studies have analyzed the impact of CET mechanisms on the electricity market. Wang et al. proposed a two-stage scheduling model to comprehensively investigate the environmental benefits of consumers participating in both electricity and CET markets [9]. Zhou et al. aimed to analyze these potential profit impacts and the possible compensation to affected generation companies through modeling the Australian National Electricity Market under a potential emission trading scheme [10]. In [11], a model was proposed to determine equilibrium fuel, power, and emission trades in an electric power supply chain framework with a load-based emission trading program. In [12], a novel model was proposed to optimize the decision-making process in the interrelated electricity, fuel, and carbon markets in order to maximize the GENCO's profit. In $[13,14]$, the short-term unit combination problem with carbon emission constraints was investigated, and a solution was developed in which the carbon emission costs will be added to the system operation costs when the total carbon emissions of a system exceed its allocated quota. Based on the classic Cournot equilibrium model, Li et al. modeled different types of units separately and established a bi-level programming model to study the impact of carbon emission costs on the equilibrium state of the market and the strategic behavior of market members [15]. Each market member can achieve the maximum benefit in this case. However, the marginal price of the electricity market can be obtained only when the independent system operator (ISO) figures out the inverse demand function of the market in advance, which is inconvenient in practice. To analyze the impact of different carbon emission quota allocation schemes on the unit combination results, system operation costs, and changes in the benefits structure, Wang et al. established a security-constrained unit combination model considering the allocation of carbon emission quotas [16]. However, it ignored the conflicts of interest between different entities of the market and failed to make a scheduling result that could take into account the different entities. Therefore, the model could not truly reflect the impact of carbon emission quotas on the marginal price of the electricity market.

This paper proposes a two-stage algorithm for calculating the locational marginal price (LMP) in the day-ahead market within the CET mechanism to analyze the impact of carbon emission allowance on the day-ahead electricity market. First, a multi-objective optimization model to minimize both system operation costs and carbon emission costs is proposed and solved by the normalized normal constraint (NNC) method. Next, a tracing model with the goal of minimizing the total costs of the power system and satisfying the constraints generated by the Pareto optimal solution is built to calculate the LMP. Finally, the proposed algorithm is applied to a real 1560-bus and 52-unit system. The impacts of different carbon emission allocation schemes on the LMP and other clearing results of the day-ahead market are analyzed. The results of the simulation show that the proposed algorithm can balance the conflicts between different generation units and reduce the total carbon emissions of the system.

The remainder of this paper is organized as follows. In Section 2, we introduce the framework and assumptions. In Section 3, we establish the two-stage model for calculating the LMP. The solution methodology is proposed in Section 4. Numerical results are presented in Section 5, and conclusions are drawn in Section 6.

\section{Market Framework and Model Assumptions}

\subsection{Market Framework}

Figure 1 shows the transaction framework in which generation units (i.e., conventional units, renewable units, and pumped storage units) participate in both the CET market and the day-ahead electricity market. During electricity generation, conventional units emit greenhouse gases, which are monitored by the government. Additionally, the government is responsible for both the allocation of 
annual carbon emission quotas to generation units at the beginning of the year and the verification of total carbon emissions to those units at the end of the year.

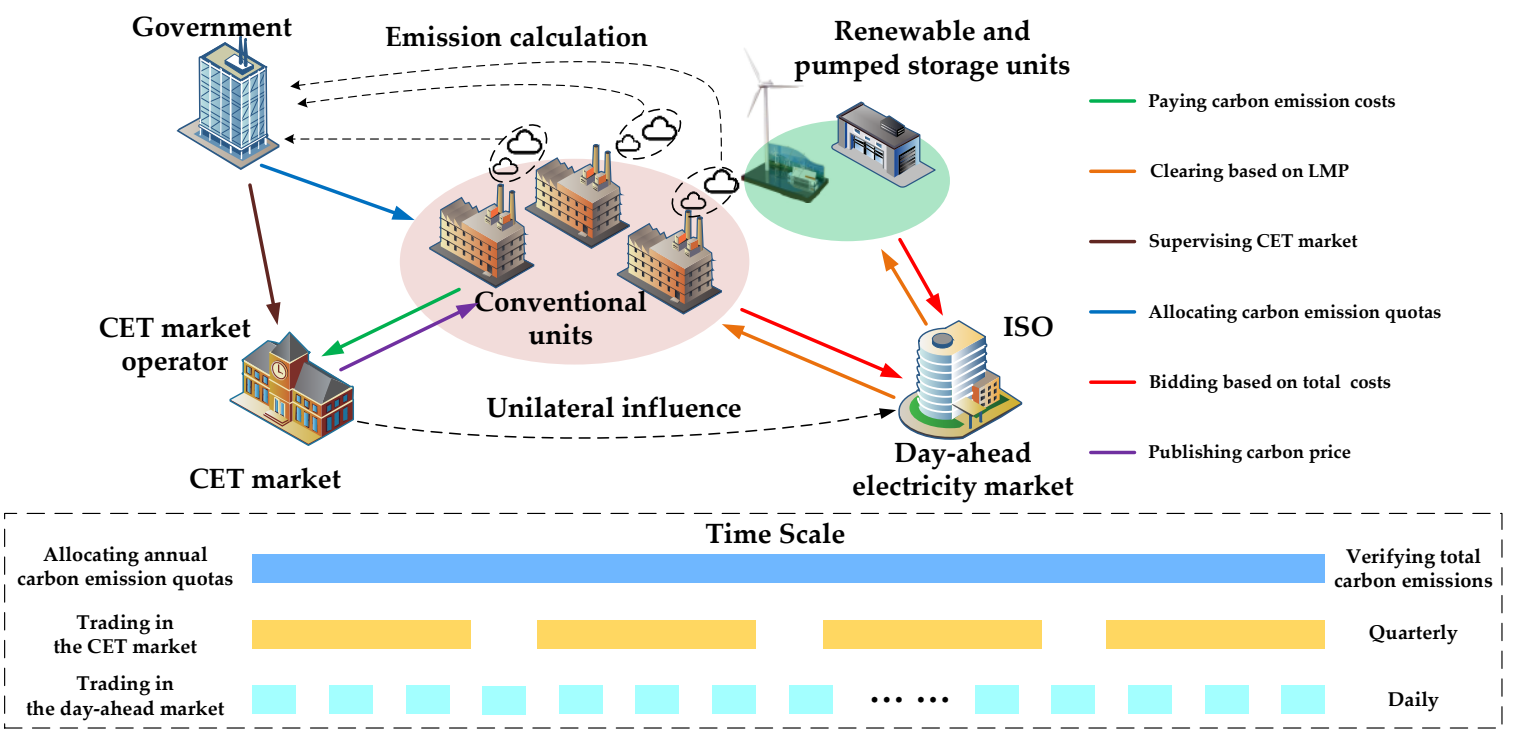

Figure 1. Market framework considering the interaction between carbon emission trading (CET) market and day-ahead electricity market.

The interaction between the CET market and day-ahead electricity market can be summarized as follows. Each generation unit offers price and capacity bidding to ISO daily within the day-ahead electricity market. Conventional units trade emission quotas in the CET market quarterly. Hence, the carbon price is relatively fixed for a certain operational day of the day-ahead electricity market. Conventional units are able to trade their surplus quotas with each other to obtain profits; however, they need to pay for carbon emission costs when their emissions exceed their prescribed free allocation quota, which will increase their generation costs. Therefore, they will evaluate the predicted carbon emission costs according to the allocated quotas obtained from the government before bidding in the day-ahead electricity market.

According to the interaction mentioned above, the CET market does have a unilateral influence on the day-ahead market within the CET mechanism, and the details are given in Section 3.

\subsection{Model Assumptions}

\subsubsection{Carbon Emission Quota Allocation Approaches}

Currently, the government allocates carbon emission quotas for various industries annually. In order to study the impact of carbon emission quotas on the marginal price of day-ahead electricity markets, this paper modifies the time scales of the quota allocation from years to days and assumes that each conventional unit can only obtain quotas from the government. The quotas are unable to deal between different units.

Before allocating carbon emission quotas for conventional units, it is necessary to specify the total amount of carbon emissions available for quota allocation. Generally, we set the benchmark by solving the unit commitment (UC) problem with CET and calculating the total carbon emissions of conventional units. Then, the total emission quota with CET can be expressed as:

$$
Q=(1-\alpha) E^{b},
$$

where $Q$ is the total daily quota available to allocate; $E^{b}$ represents the total daily emission of conventional units without CET; and $\alpha$ denotes the emission reduction factor. 
To allocate the quotas to generating units, two concrete approaches are proposed [10], including the historical carbon emission-based method and the power generation performance-based method.

(1) The historical carbon emission-based method:

The quota $E_{q, i}$ of unit $i$ is allocated based on the historical emissions quantities without CET, which can be expressed as:

$$
E_{q, i}=\frac{E_{i}^{b}}{E^{b}} Q
$$

where $E_{i}^{b}$ is the carbon emissions of unit $i$ without CET.

(2) The power generation performance-based method:

Power generation performance $G$ is the amount of $\mathrm{CO}_{2}$ emitted by conventional unit $i$ for producing one unit of electricity at time $t$, which is expressed as:

$$
G=\frac{Q}{\sum_{i=1}^{N_{1}} \sum_{t=1}^{24} P_{i, t}}
$$

where $P_{i, t}$ is the output of unit $i$ at time $t$, and $N_{1}$ represents the total number of conventional generating units.

Hence, the quota $E_{q, i}$ of the unit $i$ can be defined as:

$$
E_{q, i}=G \sum_{t=1}^{24} P_{i, t} .
$$

\subsubsection{Stepwise Bidding of Conventional Units}

In this paper, we assume that the generating units bid and offer in the day-ahead electricity market, while the consumers only declare quantity in the demand side. Without CET, the quotation curve of a conventional unit is segmented, as shown in Figure 2, where $P_{i, \min }$ and $P_{i, \max }$ represent the lower and upper output limits of unit $i$, respectively. The quotation of unit $i$ in the $k$ th step at time $t$ can be written as:

$$
C_{i . t}^{k}=a_{i}\left(P_{i, t}^{k \prime}\right)^{2}+b_{i} P_{i, t}^{k \prime}+c_{i}
$$

where $a_{i}, b_{i}$, and $c_{i}$ denote the production cost coefficients, and $P_{i, t}^{k}$ represents the bidding quantities corresponding to the quotation of unit $i$ in the $k$ th step at time $t$, which is written as:

$$
P_{i, t}^{k \prime}=\left\{\begin{array}{lc}
P_{i, \min }+L_{i} & k=1,2 \\
P_{i, \min }+2 L_{i} & k=3 \\
P_{i, \min }+3 L_{i} & k=4 \\
P_{i, \min }+4 L_{i} & k=5
\end{array},\right.
$$

where $L_{i}$ represents the length of the multi-section quotation quantity of conventional unit $i$.

In general, the sum of the winning quantity for each segment must be equal to the total output of the unit $i$ in Figure 1, that is,

$$
P_{i, t}=\sum_{k=1}^{K} P_{i, t^{\prime}}^{k}
$$

where $P_{i, t}^{k}$ represents the capacity offer in the $k$ th step by conventional unit $i$ at time $t$. Each segment of quantity needs to meet the upper and lower limits of the segment, that is,

$$
\left\{\begin{array}{l}
P_{i, t}^{k}=P_{i, \min } I_{i, t}, k=1 \\
0 \leq P_{i, t}^{k} \leq L_{i} I_{i, t}, 1<k \leq K
\end{array} .\right.
$$


Considering the mechanism of CET, conventional unit $i$ has to pay its carbon emission costs when its emissions exceed its allocation quota. Therefore, the total costs of generating unit $i$ will rise, and then unit $i$ may adjust its online quotation strategy by adding the predicted carbon emission costs, corresponding to its quotas, in each segment of the declared prices, that is,

$$
C_{i . t}^{k}=a_{i}\left(P_{i, t}^{k \prime}\right)^{2}+b_{i} P_{i, t}^{k \prime}+c_{i}+\frac{\left(\sum_{t=1}^{24} \beta_{c i} P_{i, \max }-\eta E_{q, i}\right) \times p r_{e}}{\sum_{t=1}^{24} P_{i, \max }},
$$

where $\beta_{c i}$ represents the emission coefficient of conventional unit $i$, and $p r_{e}$ is the carbon emission trading price, which we set to 15 yuan/ton.

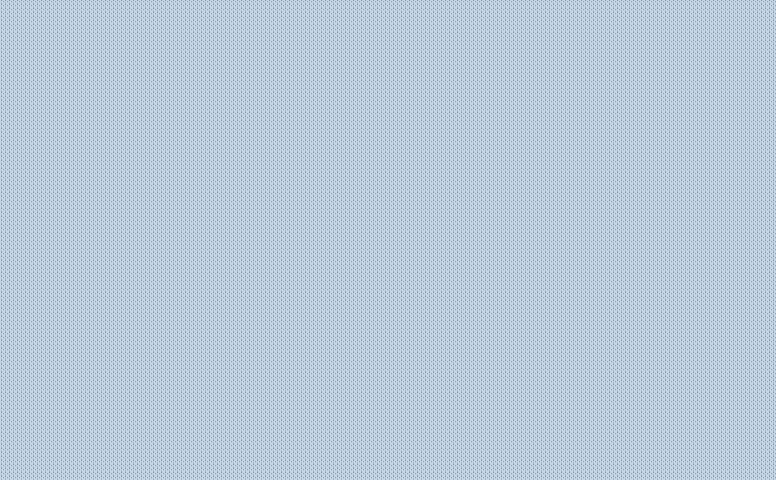

Figure 2. Multi-segment bid curve for conventional units $(K=5)$.

\section{Two-Stage Model of Locational Marginal Price Calculation with Carbon Emission Trading (CET)}

To analyze the effect of carbon emission quota allocation on the LMP of the day-ahead electricity market, a two-stage model within the CET mechanism is proposed in this paper. The relationship between the two stages of the model is shown in Figure 3.

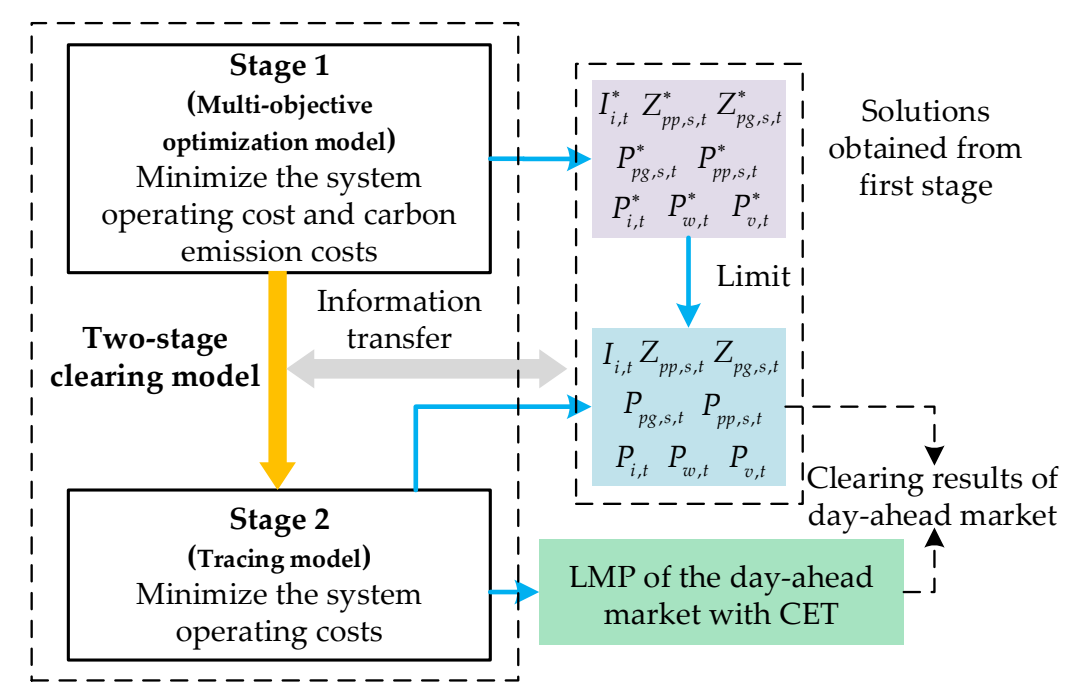

Figure 3. Relationship between the two stages of the model.

In the first stage, there is an optimization for both system operation costs and carbon emission costs to determine the outputs and statuses of the generation units. The solutions obtained from the 
first stage can balance the conflicts between different objectives but fail to analyze the impact on the LMP. We utilize these solutions to limit the variables in the second stage to make sure that the outputs and statuses of generation units are the same as those obtained from the first stage. Then, the LMP calculated in the second stage is used to analyze the impact of the CET mechanism.

\subsection{The First Stage of the Model: Mutli-Objective Optimization}

In the first stage, a multi-objective optimization model is established with the objective of simultaneously minimizing (1) the operation costs of the power system and (2) the total costs of carbon emissions paid by traditional generator units. Meanwhile, this model should satisfy the following constraints: (1) the unit's startup and shutdown cost constraints, (2) power balance constraints, (3) lower and upper output limits of conventional units, (4) ramp rate constraints, (5) minimum online and offline duration time constraints, (6) pumped storage unit's operational constraints, (7) spinning reserve constraints, (8) consistent start-stop status of gas-steam combined cycle unit, (9) lower and upper output limits of renewable units, and so on.

In this stage, we establish day-ahead scheduling to determine start-stop statuses and outputs of conventional units, outputs of renewable units, and discharging/charging statuses and outputs of pumped storage units.

\subsubsection{Objectives}

The first objective function $J_{1}(\cdot)$ is defined as the operation costs of the power system, including the costs of electricity purchased from generating units, the costs of spinning reserve, the costs of the unit's startup and shutdown, and the unit no-load costs, which can be expressed as follows:

$$
\begin{aligned}
\min J_{1}= & \sum_{t=1}^{24}\left\{\sum _ { i = 1 } ^ { N _ { 1 } } \left[\sum_{k=1}^{K} C_{i, t}^{k} P_{i, t}^{k}+C_{i, t}^{U} s_{u i, t}+C_{i, t}^{D} s_{d i, t}\right.\right. \\
& \left.\left.+C_{i}^{N L} I_{i, t}+C_{i U, t}+C_{i D, t}\right]+\sum_{s=1}^{N_{2}}\left(C_{s, t}^{U} s_{u s, t}+C_{s, t}^{D} s_{d s, t}+C_{s U, t}+C_{s D, t}\right)\right\}^{\prime}
\end{aligned}
$$

where $C_{i, t}^{U}$ and $C_{i, t^{\prime}}^{D}$ respectively, denote the upward and downward reserve prices bid by conventional unit $i$ at time $t ; s_{u i, t}$ and $s_{d i, t}$, respectively, represent the upward and downward reserve capacities offered by conventional unit $i$ at time $t ; C_{i U, t}$ and $C_{i D, t}$, respectively, denote the startup and shutdown costs of the conventional unit $i$ at time $t ; C_{s, t}^{U}$ and $C_{s, t}^{D}$, respectively, represent the upward and downward reserve prices bid by pumped storage unit $s$ at time $t ; s_{u s t}$ and $s_{d s, t}$, respectively, represent the upward and downward reserve capacities offer by pumped storage unit $s$ at time $t ; C_{s U, t}$ and $C_{s D, t}$, respectively, denote the startup and shutdown costs of the pumped storage unit $s$ at time $t ; K$ is the number of quotation segments for conventional units; and $C_{i, t}^{k}$ represents the price bid in the $k$ th step by conventional unit $i$ at time $t$. In this model, we mandate that the pumped storage unit and renewable unit take a zero quotation in the day-ahead market to ensure they win the bid first.

The second objective function $J_{2}(\cdot)$ is defined as the total cost of carbon emissions paid by traditional generator units as follows:

$$
\min J_{2}=\sum_{i=1}^{N_{1}} E_{i, c} \times p r_{e}
$$

where $E_{i, c}$ represents the overall emissions exceeding the quota of conventional unit $i$ in a day, which can be expressed as follows:

$$
E_{i, c}=\sum_{t=1}^{24} E_{i, t}-\eta E_{q, i},
$$

where $\eta$ denotes the rate of free quota; and $E_{i, t}$ is the emissions of conventional unit $i$ at time $t$, which can be expressed as follows:

$$
E_{i, t}=\beta_{c i} P_{i, t} .
$$


3.1.2. Unit's Startup and Shutdown Cost Constraints

$$
\begin{gathered}
\left\{\begin{array}{lc}
C_{i U, t} \geq K_{i}\left(I_{i, t}-I_{i, t-1}\right), & C_{i U, t} \geq 0 \\
C_{s U, t} \geq K_{s}\left(Z_{s, t}-Z_{s, t-1}\right), & C_{s U, t} \geq 0
\end{array},\right. \\
\left\{\begin{array}{lr}
C_{i D, t} \geq J_{i}\left(I_{i, t-1}-I_{i, t}\right), & C_{i D, t} \geq 0 \\
C_{s D, t} \geq J_{s}\left(Z_{s, t-1}-Z_{s, t}\right), & C_{s D, t} \geq 0
\end{array},\right.
\end{gathered}
$$

where $K_{i}$ and $J_{i}$ represent the startup and shutdown cost coefficients of the conventional unit $i$, respectively; $K_{s}$ and $J_{s}$ represent the startup and shutdown cost coefficients of the pumped storage unit $s$, respectively; and $I_{i, t}$ and $Z_{s, t}$ denote the on/off statuses of the conventional unit $i$ and the discharging/charging statuses of the pumped storage unit $s$ at time $t$, respectively.

3.1.3. Lower and Upper Limits of Conventional Units

$$
I_{i, t} P_{i, \min } \leq P_{i, t} \leq I_{i, t} P_{i, \max }
$$

3.1.4. Ramp Rate Constraints of Conventional Units

$$
\left\{\begin{array}{l}
P_{i, t}-P_{i, t-1} \leq r_{u i} T_{60} I_{i, t-1}+P_{i, \min }\left(I_{i, t}-I_{i, t-1}\right) \\
P_{i, t-1}-P_{i, t} \leq r_{d i} T_{60} I_{i, t}+P_{i, \min }\left(I_{i, t-1}-I_{i, t}\right)
\end{array},\right.
$$

where $r_{u i}$ and $r_{d i}$ represent the upward and downward ramping rate limits of unit $i$, respectively.

3.1.5. Pumped Storage Unit's Operational Constraints

$$
\begin{gathered}
\left\{\begin{array}{c}
0 \leq P_{p g, s, t} \leq P_{p g, s, \max } \cdot Z_{p g, s, t} \\
P_{p p, s, \max } \cdot Z_{p p, s, t} \leq P_{p p, s, t} \leq 0
\end{array},\right. \\
Z_{s, t}=Z_{p g, s, t}+Z_{p p, s, t} \leq 1, \\
\sum_{t=1}^{T} P_{p g, s, t}+\xi \cdot \sum_{t=1}^{T} P_{p p, s, t}=0,
\end{gathered}
$$

where $P_{p g, s, t}$ and $P_{p p, s, t}$ denote the discharging and charging power of the pumped storage unit $s$ at time $t$, respectively; $P_{p g, s, \max }$ and $P_{p p, s, \max }$ represent the upper limits of discharging and charging power of unit s, respectively; $Z_{p g, s, t}$ and $Z_{p p, s, t}$ indicate the discharging/charging status of the pumped storage unit $s$, respectively; and $\xi$ is the conversion efficiency of the pumped storage unit, which is set to $75 \%$.

3.1.6. Spinning Reserve Constraints

$$
\begin{gathered}
\left\{\begin{array}{c}
S_{u, t}=\sum_{i=1}^{N_{1}+N_{2}} s_{u i, t} \geq P_{\text {Load }, t} \cdot L_{u} \% \\
s_{d, t}=\sum_{i=1}^{N_{1}+N_{2}} s_{d i, t} \geq P_{\text {Load }, t} \cdot L_{d} \%
\end{array},\right. \\
\left\{\begin{array}{l}
0 \leq s_{u s, t} \leq P_{p g, s, \max } Z_{p g, s, t}-P_{p g, s, t}-P_{p p, s, t} \\
0 \leq s_{d s, t} \leq P_{p g, s, t}+P_{p p, s, t}-P_{p p, s, \max } Z_{p p, s, t}
\end{array},\right. \\
\left\{\begin{array}{l}
0 \leq s_{u i, t} \leq \min \left(P_{i, \max } \cdot I_{i, t}-P_{i, t}, r_{u i} \cdot T_{60} \cdot I_{i, t}\right) \\
0 \leq s_{d i, t} \leq \min \left(P_{i, t}-P_{i, \min } \cdot I_{i, t}, r_{d i} \cdot T_{60} \cdot I_{i, t}\right)
\end{array},\right.
\end{gathered}
$$


where $S_{u, t}$ and $S_{d, t}$ indicate the capacity of the upward and downward spinning reserve requirements at time $t$, respectively; and $L_{\mathcal{u}} \%$ and $L_{d} \%$ denote the coefficients of load forecast deviation for the upward and downward reserve capacity of the system, respectively.

3.1.7. Lower and Upper Output Limits of Renewable Units

$$
\left\{\begin{array}{l}
0 \leq P_{v, t} \leq P_{v, t, \max } \\
0 \leq P_{w, t} \leq P_{w, t, \max }
\end{array},\right.
$$

where $P_{w, t}$ and $P_{v, t}$ represent the output of the wind farm unit $w$ and the photovoltaic power generation unit $v$ at time $t$, respectively.

\subsubsection{Power Balance Constraints}

$$
\sum_{i=1}^{N_{1}} P_{i, t}+\sum_{s=1}^{N_{2}}\left(P_{p g, s, t}+P_{p p, s, t}\right)+\sum_{j=1}^{N_{3}} P_{j, t}+\sum_{w=1}^{N_{4}} P_{w, t}+\sum_{v=1}^{N_{5}} P_{v, t}=\sum_{d=1}^{N_{\mathrm{D}}} P_{d, t}+P_{\text {Loss }, t},
$$

where $P_{j, t}$ is the injected power of the tie line unit $j$ at time $t ; P_{d, t}$ denotes the demand of load bus $d$ at time $t$; and $P_{\text {Loss, } t}$ indicates the power loss of the system at time $t$, which is set to $0.075 \%$ of the total load demand.

3.1.9. Network Transmission Constraints

$$
\begin{gathered}
P_{l, t}=\sum_{i=1}^{N_{1}} G_{l, i} P_{i, t}+\sum_{s=1}^{N_{2}} G_{l, s}\left(P_{p g, s, t}+P_{p p, s, t}\right)+\sum_{j=1}^{N_{3}} G_{l, j} P_{j, t}+\sum_{w=1}^{N_{4}} G_{l, w} P_{w, t}+\sum_{v=1}^{N_{5}} G_{l, v} P_{v, t}-\sum_{d=1}^{N_{\mathrm{D}}} D_{l, d} P_{d, t} . \\
-P_{l \max } \leq P_{l, t} \leq P_{l \max } l=1,2, \ldots, N_{L} ; t=1,2, \ldots, T .
\end{gathered}
$$

3.1.10. Minimum Online and Offline Duration Time Constraints

$$
\begin{aligned}
& \left\{\begin{array}{l}
I_{i, t}=1, t \in\left[1, U_{i}\right], U_{i}=\min \left\{T,\left(T_{\text {on }_{\_} i}-X_{\text {on } \_i, 0}\right) I_{i, 0}\right\} \\
t+T_{\text {on_ } \_}-1 \\
\sum_{n=t} I_{i, n} \geq T_{\text {on_i }}\left(I_{i, t}-I_{i, t-1}\right), t \in\left[U_{i}+1, T-T_{\text {on_i }}+1\right] \\
\sum_{n=t}^{T}\left[I_{i, n}-\left(I_{i, t}-I_{i, t-1}\right)\right] \geq 0, t \in\left[T-T_{\text {on_i }}+2, T\right]
\end{array}\right. \\
& \left\{\begin{array}{l}
I_{i, t}=0, t \in\left[1, D_{i}\right], D_{i}=\min \left\{T,\left(T_{o f f_{-} i}-X_{o f f_{-} i, 0}\right)\left(1-I_{i, 0}\right)\right\} \\
t+T_{\text {off } \_}-1 \\
\sum_{n=t}\left(1-I_{i, n}\right) \geq T_{o f f_{-} i}\left(I_{i, t-1}-I_{i, t}\right), \\
t \in\left[D_{i}+1, T-T_{o f f_{-} i}+1\right] \\
\sum_{n=t}^{T}\left[1-I_{i, n}-\left(I_{i, t-1}-I_{i, t}\right)\right] \geq 0, t \in\left[T-T_{o f f_{-} i}+2, T\right]
\end{array}\right.
\end{aligned}
$$

where $U_{i} / D_{i}$ represents the number of hours unit $i$ must be initially on/off due to its minimum on/off time limit; $T_{\text {on } \_} / T_{\text {off } \_i}$ represents the minimum on/off time of unit $i$; and $X_{\text {on } \_i, 0} / X_{\text {off } \_i, 0}$ represents the initial on/off hours of unit $i$.

3.1.11. Consistent Start-Stop Status Constraint of Gas-Steam Combined Cycle Units

$$
I_{i, t}=I_{j, t},
$$


where $I_{i, t}$ and $I_{j, t}$ denote the on/off status of a large-capacity unit $i$ and a small-capacity unit $j$, respectively. In the proposed model, we separately dispatch two different units of a gas-steam combined cycle unit and control their start-stop states to be consistent.

\subsubsection{Simplified Formation of the First Stage of the Model}

For the convenience of later discussion, we rewrite the multi-objective optimization model (10)-(30) as the following compact form:

$$
\begin{gathered}
\min \left(J_{1}, J_{2}\right), \\
\text { s.t. } \quad f\left(x_{1}, x_{2}\right)=0, \\
\mathrm{~g}\left(x_{1}, x_{2}\right) \leq 0,
\end{gathered}
$$

where $x_{1}$ represents the vector of outputs of conventional units, pumped storage units, and renewable units; and $x_{2}$ denotes the vector of the on/off status of the conventional units and the discharging/charging status of pumped storage units.

\subsection{The Second Stage of the Model: Tracking Pareto Optimal Solution}

The day-ahead market-clearing model is essentially a security-constrained unit commitment problem [17]. Therefore, the analytic expression of the marginal electricity price in the day-ahead market can be obtained using information such as the Lagrangian multipliers of the power balance constraints. In the first stage, we strike a better balance among the conflicts between different objectives but fail to obtain the marginal electricity price information directly. In the second stage, we propose a typical economic dispatch model to avoid reducing the convexity of the clearing problem for calculating the LMP [18]. We utilize the solutions obtained from the first stage of the model to fix the on/off status of the conventional units and the discharging/charging status of the pumped storage units as well as to limit the outputs of those generation units.

\subsubsection{Objective}

The objective of the second stage is to minimize the costs of system operation with the fixed on/off status of the conventional units and the fixed discharging/charging status of the pumped storage units, which can be written as Equation (34).

$$
\min J=\sum_{t=1}^{24}\left\{\sum_{i=1}^{N_{1}}\left[\sum_{k=1}^{K} C_{i, t}^{k} P_{i, t}^{k}+C_{i, t}^{U} s_{u i, t}+C_{i, t}^{D} s_{d i, t}\right]+\sum_{s=1}^{N_{2}}\left(C_{s, t}^{U} s_{u s, t}+C_{s, t}^{D} s_{d s, t}\right)\right\} .
$$

\subsubsection{Constraints}

The second stage of the model should satisfy not only the constraints (16)-(18) and (20)-(27) mentioned in the first stage but also the additional constraints (35)-(39) listed below, which can ensure the solution obtained from the second stage of the model is the same as that obtained from the first stage:

$$
\begin{gathered}
P_{i, t}^{*}-\varepsilon \leq P_{i, t} \leq P_{i, t}^{*}+\varepsilon, \\
P_{w, t}^{*}-\varepsilon \leq P_{w, t} \leq P_{w, t}^{*}+\varepsilon, \\
P_{v, t}^{*}-\varepsilon \leq P_{v, t} \leq P_{v, t}^{*}+\varepsilon, \\
\left(P_{p g, s, t}^{*}+P_{p p, s, t}^{*}\right)-\varepsilon \leq P_{p g, s, t}+P_{p p, s, t}, \\
P_{p g, s, t}+P_{p p, s, t} \leq\left(P_{p g, s, t}^{*}+P_{p p, s, t}^{*}\right)+\varepsilon,
\end{gathered}
$$


where $P_{i, t^{\prime}}^{*} P_{w, t^{\prime}}^{*} P_{v, t^{\prime}}^{*}$ and $\left(P_{p g, s, t}^{*}+P_{p p, s, t}^{*}\right)$ represent the output of the conventional unit $i$, the wind farm $w$, the PV power generation $v$, and the pumped storage unit $s$ at time $t$, obtained from the first stage model, respectively; $\varepsilon$ denotes the relaxation error and is set to 0.0000001 .

In addition, the LMP for the settlement [19] is expressed as follows:

$$
L M P_{k, t}=\lambda_{t}-\sum_{l=1}^{N_{L}}\left(\tau_{l, t}^{\max }-\tau_{l, t}^{\min }\right) G_{l, k}
$$

where $L M P_{k, t}$ is the marginal price of the node $k$ at time $t ; \lambda_{t}$ represents the Lagrangian multipliers of the power balance constraints at time $t ; \tau_{l, t}^{\max }$ and $\tau_{l, t}^{\min }$ denote the Lagrangian multipliers of the maximum forward power flow constraint and the maximum backward power flow constraint for line $l$ at time $t$, respectively; and $G_{l, k}$ indicates the transfer distribution factor of generator output from generator node $k$ to line $l$.

According to Equation (40), if transmission is constraining, the LMPs at the two ends of the congested line are different.

\section{Solution and Methodology}

In this section, we discuss the optimization algorithm to solve the two-stage model. Obviously, the second stage of the model is a linear programming problem and can be easily solved using the GAMS/CPLEX solver [20], whereas the first stage of the model in (31)-(33) is a multi-objective mixed-integer linear programming problem.

We apply the NNC method [21] to solve the multi-objective mixed-integer linear programming problem, which is elaborated as follows.

Step 1: Solve the single objective model, which aims to minimize $J_{1}$ and is subject to (32) and (33), to obtain the minimum value of objective $J_{1}$, written as $J_{1} \mathrm{~min}$, and the maximum value of objective $J_{2}$, written as $J_{2} \max$.

Step 2: Solve the single objective model, which aims to minimize $J_{2}$ and is subject to (32) and (33), to obtain the minimum value of objective $J_{2}$, written as $J_{2} \mathrm{~min}$, and the maximum value of objective $J_{1}$, written as $J_{1 \text { max }}$.

Step 3: Normalize the objective (31) by $J_{1 \min }, J_{1 \max }, J_{2 \min }$, and $J_{2 \max }$ obtained in Steps 1 and 2, that is,

$$
\bar{J}=\left[\bar{J}_{1}, \bar{J}_{2}\right]=\left[\frac{J_{1}-J_{1 \min }}{J_{1 \max }-J_{1 \min }}, \frac{J_{2}-J_{2 \min }}{J_{2 \max }-J_{2 \min }}\right] .
$$

Step 4: Take $\bar{J}_{1}$ as the abscissa, $\bar{J}_{2}$ as the ordinate, and $A_{1}(0,1)$ and $A_{2}(1,0)$ as the anchor points, and depict the Pareto front, Utopia line, and normalized objective space in Figure 4.

Step 5: Divide the Utopia line into $m$ equal segments, and generate $m+1$ evenly distributed segmentation points in the Utopia line, that is,

$$
\begin{aligned}
& \bar{X}_{p j}=\left(1-\frac{j}{m}\right)(0,1)+\frac{j}{m}(1,0)=\left(\frac{j}{m}, 1-\frac{j}{m}\right) . \\
& j=0,1, \ldots \ldots, m
\end{aligned}
$$

Step 6: Draw the normal $\vec{P}_{j}$ of the Utopia line across $\bar{X}_{p j}$, and generate a corresponding Pareto point $B_{j}$ by intersecting this normal with the Pareto front. In order to find the optimal point $B_{j}$ in the Pareto front, minimize $\bar{J}_{2}$, which is subject to Equations (32) and (33). This optimization process should be forced to stay within the feasible region of the normalized objective space, i.e., the region labeled with the dot in Figure 4. Mathematically, the single objective optimization problem can be expressed as follows:

$$
\begin{gathered}
\min \bar{J}_{2}, \\
\text { s.t. } \quad f\left(x_{1}, x_{2}\right)=0,
\end{gathered}
$$




$$
\begin{gathered}
\mathrm{g}\left(x_{1}, x_{2}\right) \leq 0, \\
\vec{N}_{1}\left(\bar{J}-\bar{X}_{p j}\right)^{T} \leq 0,
\end{gathered}
$$

where $\bar{J}=\left(\bar{J}_{1}, \bar{J}_{2}\right)$ represents the point in normalized objective space; Equation (45) is an additional inequality to force the upper left region of $\vec{P}_{j}$ to be feasible, as denoted with the dot in Figure 4; and the vector $\vec{N}_{1}=1 \angle-45^{\circ}$ denotes the direction from $\mathrm{A}_{1}$ to $\mathrm{A}_{2}$.

In this paper, we use the GAMS/CPLEX solver to solve the single objective optimization model in Equations (42)-(45), which is a mixed-integer linear programming problem. When $\vec{P}_{j}$ moves along the Utopia line from $\mathrm{A}_{1}$ to $\mathrm{A}_{2}$ by setting $j=1,2, \ldots, m-1$, the Pareto points except for the endpoints are obtained by solving the above single objective optimization problems.

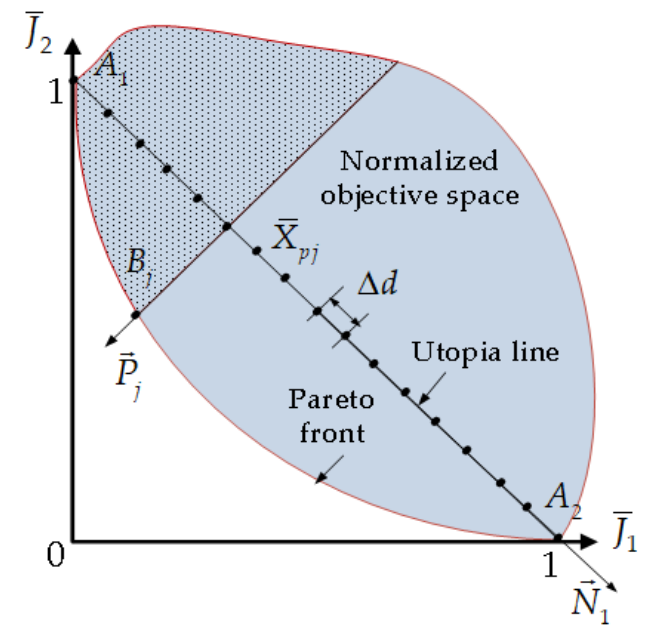

Figure 4. Pareto front, Utopia line, and normalized objective space.

\section{Case Studies and Numerical Results}

\subsection{Overview of Test System}

This study proceeds with calculations using the operation data of a real 1560-bus system in China to verify the performance of the proposed methodology. This system consists of 729 branches, 1028 transformer branches, and 52 generators, including four nuclear power plants with total capacities of $4152 \mathrm{MW}$, whose daily outputs are constant; two pumped storage units with a capacity of $350 \mathrm{MW}$; two wind farms with capacities of $360 \mathrm{MW}$ and $280 \mathrm{MW}$, respectively; two PV units with capacities of $250 \mathrm{MW}$ and $220 \mathrm{MW}$, respectively; four refuse incineration power plants; six coal-fired units; twenty-eight gas-fired units; and four combined heat and power units (all gas-fired). The total installed capacity of the system is 11,543 MW. This system has peak and valley loads of 17,434 MW and $9370 \mathrm{MW}$, respectively. Figure 5 presents the forecast total daily load, the outputs of two wind farms, and the outputs of two PV units. For this system, $L_{u} \%$ and $L_{d} \%$ are set to $3 \%$ and $1 \%$, respectively; $\eta$ is set to $95 \%$; and the emission coefficients of coal-fired and gas-fired units are set to $0.85 \mathrm{t} / \mathrm{MWh}$ and $0.35 \mathrm{t} / \mathrm{MWh}$ [22], respectively. 


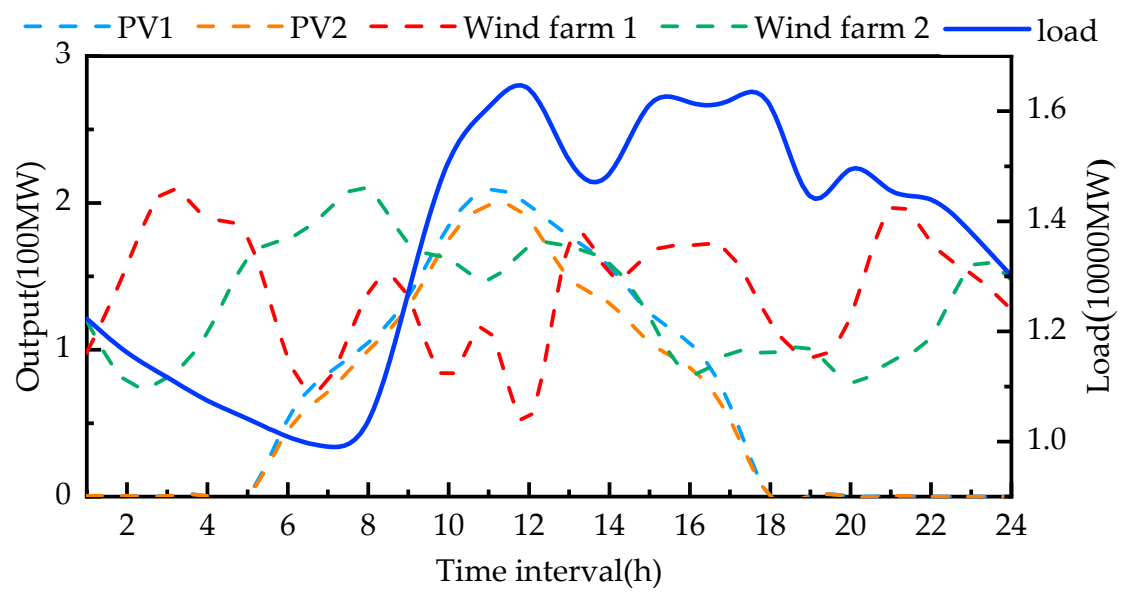

Figure 5. Forecast total daily load, the outputs of two wind farms, and the outputs of two PV units.

\subsection{Normalized Normal Constraint (NNC) Method for Solving the First Stage of the Model}

First, we use the historical carbon emission-based method to allocate carbon emission quotas for the conventional units. According to the NNC method mentioned above, we can obtain several optimal solutions from the first stage of the model with minimum system operation costs and minimum carbon emission costs. In the simulation, we set $m$ as 10, which indicates that the Utopia line is divided into 10 segments. Then, we can use the GAMS/CPLEX solver to obtain the Pareto front. Table 1 and Figure 6 illustrate the 11 optimal solutions of the Pareto front, representing the costs of system operation and the costs of carbon emission.

Table 1. Total system operation costs and total carbon emission costs represented by 11 Pareto frontier points.

\begin{tabular}{ccc}
\hline$j$ & $\bar{J}_{1}$ & $\boldsymbol{J}_{2}$ \\
\hline 0 & $-2.38 \times 10^{-15}$ & 1 \\
\hline 1 & $1.37 \times 10^{-4}$ & 0.80016 \\
\hline 2 & $4.09 \times 10^{-4}$ & 0.60041 \\
\hline 3 & 0.00116 & 0.40216 \\
\hline 4 & 0.01083 & 0.21083 \\
\hline 5 & 0.05253 & 0.05253 \\
\hline 6 & 0.2 & $5.29 \times 10^{-15}$ \\
\hline 7 & 0.4 & $3.66 \times 10^{-15}$ \\
\hline 8 & 0.6 & $4.88 \times 10^{-15}$ \\
\hline 9 & 0.8 & $3.66 \times 10^{-15}$ \\
\hline 10 & 1 & $1.22 \times 10^{-15}$ \\
\hline
\end{tabular}




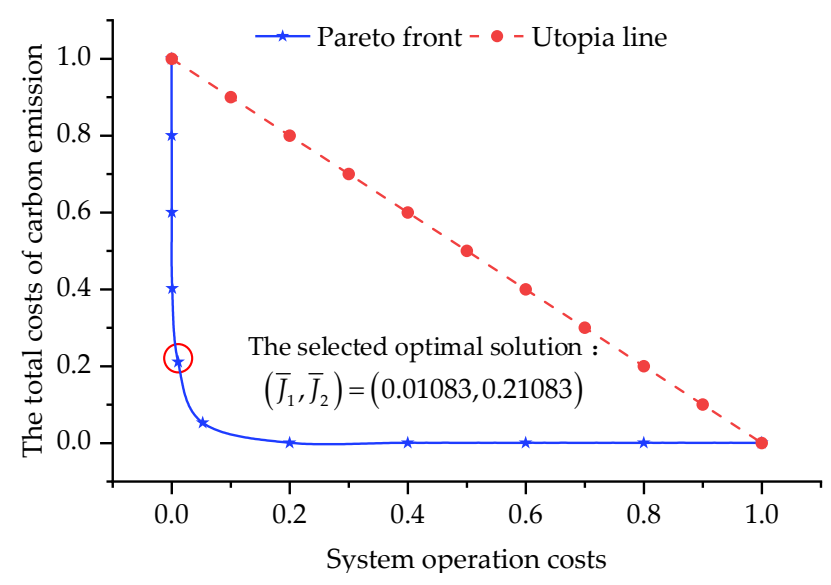

Figure 6. Pareto front and optimal compromise solution.

According to Table 1 , the value of $j$ varies from 0 to 10, indicating that the weights of the objectives $\bar{J}_{1}$ and $\bar{J}_{2}$ change accordingly. After obtaining the optimal solution set, we need to select the compromise solution to fix the on/off statuses and outputs of generating units. Various techniques to select the compromise solution were proposed in $[23,24]$. In this paper, we expect to find a compromised optimal solution that minimizes the sacrifice to $J_{1}$ while greatly reducing the value of $J_{2}$. Therefore, we can find that the most suitable solution is at $j=4$, according to the comparison between the nodes of the Pareto front and their values corresponding to objectives $\bar{J}_{1}$ and $\bar{J}_{2}$ in Table 1 and Figure 4 . With this solution, the values of $\bar{J}_{1}$ and $\bar{J}_{2}$ are 0.01083 and 0.21083 , respectively, indicating that the system operation costs are very close to the minimum, and the total carbon emission costs paid by conventional units are small. Hence, we choose the point $\left(\bar{J}_{1}, \bar{J}_{2}\right)=(0.01083,0.21083)$ as the compromise solution.

The traditional clearing model for calculating the LMP only minimizes the system operation costs, which will greatly reduce the control of carbon emissions by the CET mechanism. To explain the advantage of using the proposed two-stage model, we first conduct a simulation in the real 1560-bus and 52-unit system with two scenarios, including clearing without the CET mechanism and clearing in the CET mechanism with the traditional model. As shown in Table 2, we can see that the total carbon emissions remain high in the CET mechanism with the traditional clearing model compared with the scenario without the CET mechanism, meaning it does little to reduce emissions.

Table 2. Comparison of total system carbon emissions under different clearing methods.

\begin{tabular}{cc}
\hline Scenario & Total Carbon Emissions (tons) \\
\hline Clearing without CET mechanism & $198,259.35$ \\
\hline Clearing in CET mechanism with traditional model & $191,733.25$ \\
\hline
\end{tabular}

In order to illustrate the performance of the proposed methodology, we compare the system operation costs and carbon emission costs obtained from the two-stage model with those of the traditional clearing model that does not consider carbon emission costs in the CET mechanism. According to Table 3, the system operation costs of the two-stage model are only $0.68 \%$ higher than those of the traditional clearing model, but the total carbon emission costs paid by conventional units are reduced by $67.25 \%$, and the carbon emissions are reduced by $20.82 \%$. Therefore, although the proposed method does not reduce the system operation costs, it greatly reduces the carbon emission costs paid by conventional units, thus alleviating conflicts between different objectives and effectively reducing system carbon emissions. 
Table 3. Comparison of results between two optimization models.

\begin{tabular}{lccc}
\hline & Traditional Clearing Model & Two-Stage Model & Rangeability \\
\hline System operation costs (¥) & $28,650,271.92$ & $28,845,093.77$ & $0.68 \%$ \\
\hline Carbon emission costs (¥) & $3,045,165.34$ & $997,291.65$ & $-67.25 \%$ \\
\hline Carbon emissions (tons) & $191,733.25$ & $151,814.39$ & $-20.82 \%$ \\
\hline
\end{tabular}

\subsection{Calculation of Locational Marginal Price (LMP) Considering the Impact of Carbon Emission Quotas}

In the simulation, we use the two-stage model to solve the LMP of the day-ahead electricity market considering the impact of carbon emission quotas. In order to ensure the accuracy of the LMP calculated by the two-stage model, we compare the solutions obtained from the first stage of the model and the second stage of the model. Figure 7 shows the comparison of outputs of renewable energy units and pumped storage units. Figure 8 illustrates the comparison of outputs of the coal-fired unit with a capacity of $350 \mathrm{MW}$.

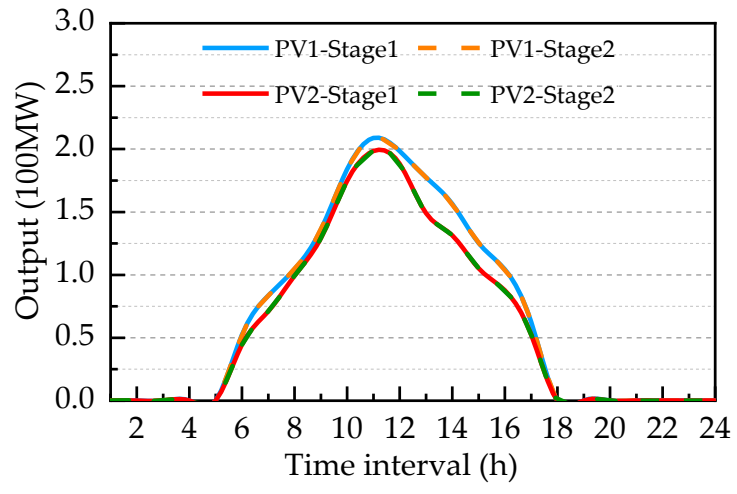

(a)

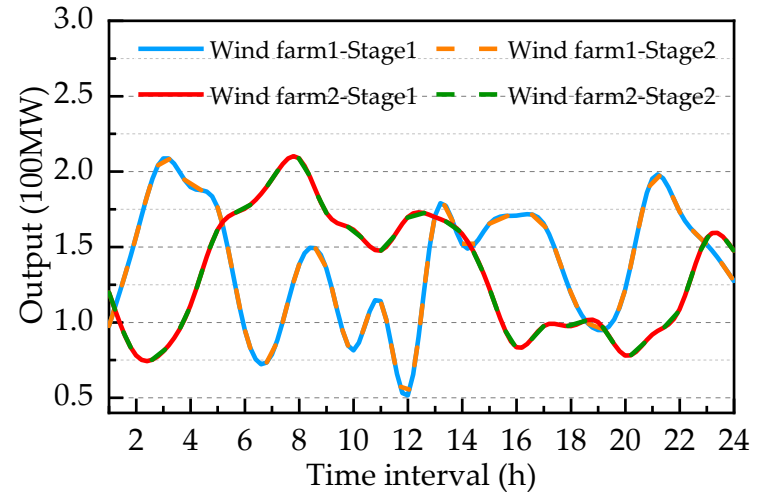

(b)
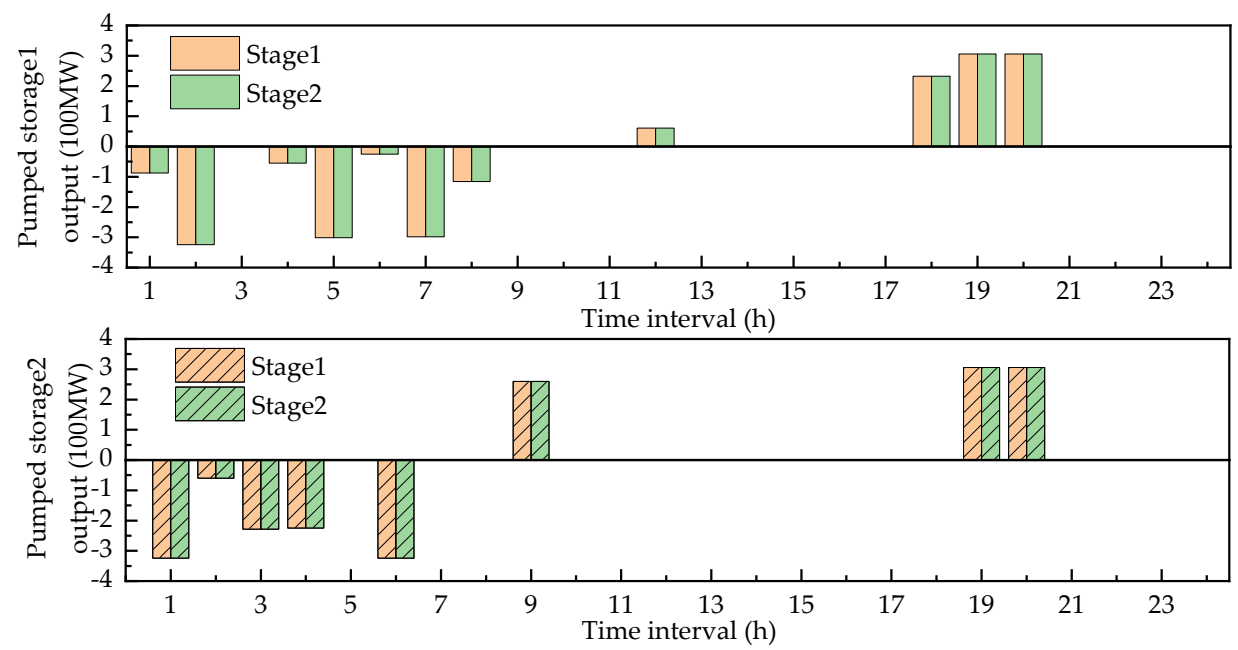

(c)

Figure 7. Comparison of outputs of renewable energy units and pumped storage units. (a) Comparison of outputs of PV units; (b) Comparison of outputs of wind farms; (c) Comparison of outputs of pumped storage units. 


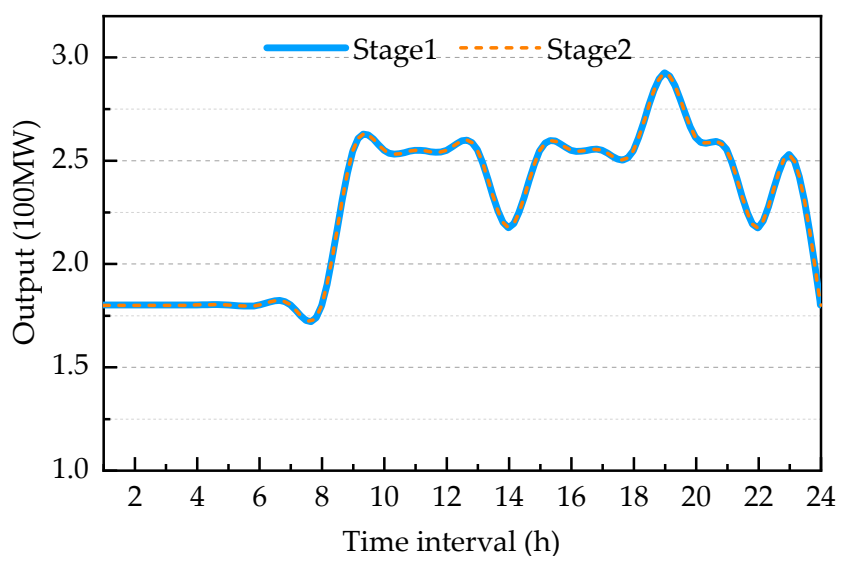

Figure 8. Comparison of outputs of the coal-fired unit with capacity of $350 \mathrm{MW}$.

Figures 7 and 8 indicate that the output of each unit in the second stage is consistent with that in the first stage. Simultaneously, the system operation costs and carbon emission costs obtained from the two stages in Table 4 are considerably close to each other. In addition, the relative errors do not exceed $10^{-7}$. Therefore, we can ensure the accuracy of the LMP obtained from the two-stage model.

Table 4. Comparison of the system operation costs and carbon emission costs obtained from the two stages of the proposed model.

\begin{tabular}{ccc}
\hline & System Operation Costs (¥) & Carbon Emission Costs (¥) \\
\hline First stage of model & $28,845,093.77$ & $997,291.65$ \\
Second stage of model & $28,845,093.44$ & $997,291.78$ \\
Error & $-0.000001141 \%$ & $0.000012677 \%$ \\
\hline
\end{tabular}

We compare the LMP calculated by the two-stage model in the CET mechanism with that obtained from the traditional clearing model without CET in the past and evaluate the impact of carbon emission quotas on the clearing of the day-ahead electricity market. In this section, we take the calculation results without CET as the benchmark. Figures 9 and 10 illustrate the comparison of the LMP.

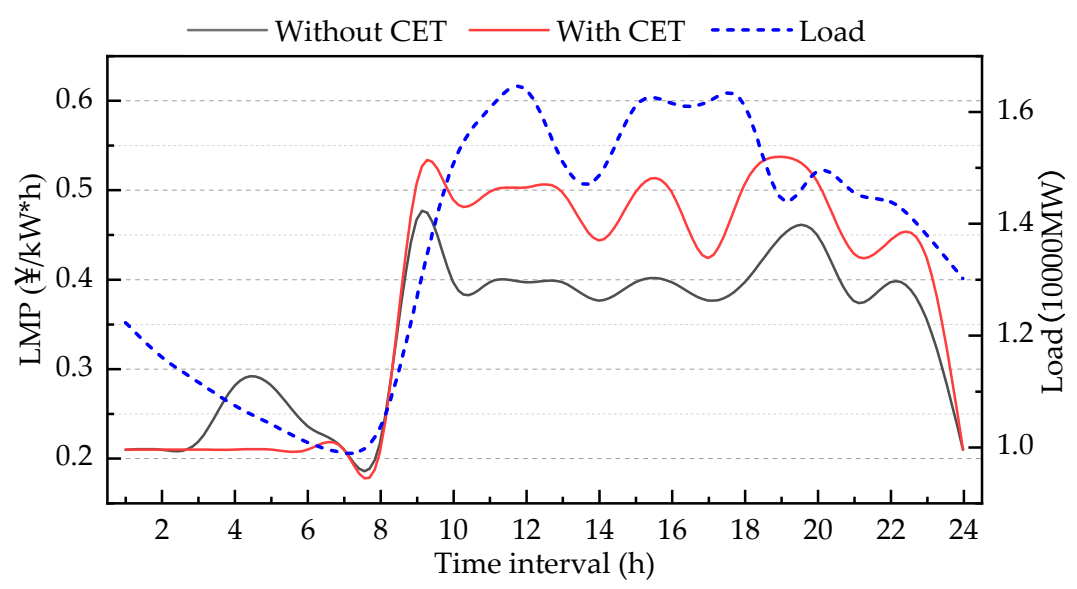

Figure 9. Comparison of the marginal price of the day-ahead market. 


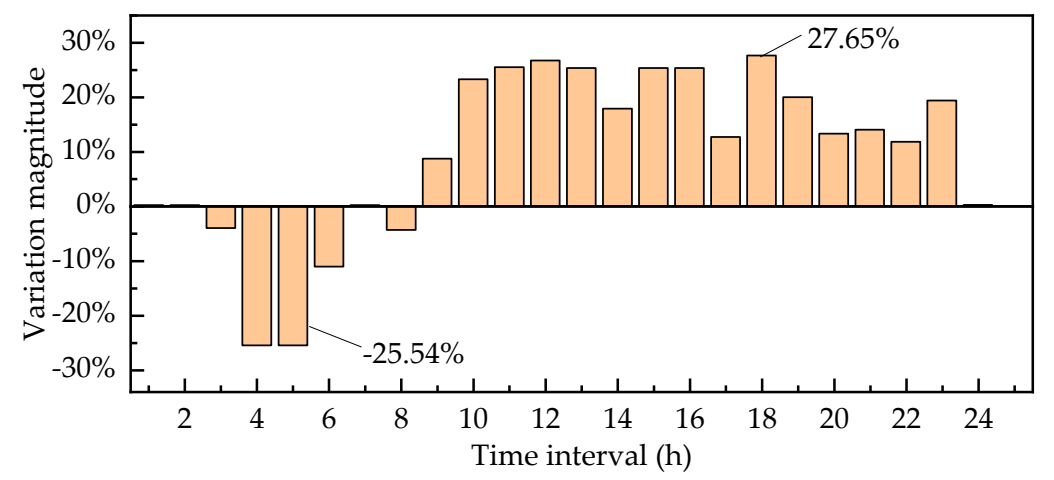

Figure 10. Variation magnitude of marginal price of the day-ahead electricity market.

According to Figures 9 and 10, the LMP has changed after considering the impacts of the CET mechanism. The LMP in the CET mechanism during the peak load period is higher than that without the impact of carbon emission quota allocation, with a maximum increase of $27.65 \%$, while it drops during the valley load period with a maximum decrease of $25.54 \%$. Both daily LMP variation trends are the same as the load curve.

\subsection{Clearing Results of Different Carbon Emission Quota Allocation Methods}

Figure 11 illustrates the comparison of carbon emissions of conventional units between two different carbon emission quota allocation methods. The on/off statuses of conventional units are the same under two different allocation methods. As shown in Figure 9, units 1-6 are coal-fired units, and units 7-26 are gas-fired units. When the quota is allocated based on the historical carbon emissions, all of the carbon emissions of the coal-fired units do not exceed their free quotas, and only three of the twenty gas-fired units are below the allocated quotas. In the case of using the power generation performance-based method to allocate quotas, the carbon emissions of some coal-fired units do exceed their reduced quotas due to their lower power generation performance. On the contrary, only six of the twenty gas-fired units have exceeded their quotas because of their increased quotas and their low carbon emission coefficients. The possibility that the carbon emissions of gas-fired units will exceed their quotas has been greatly reduced.
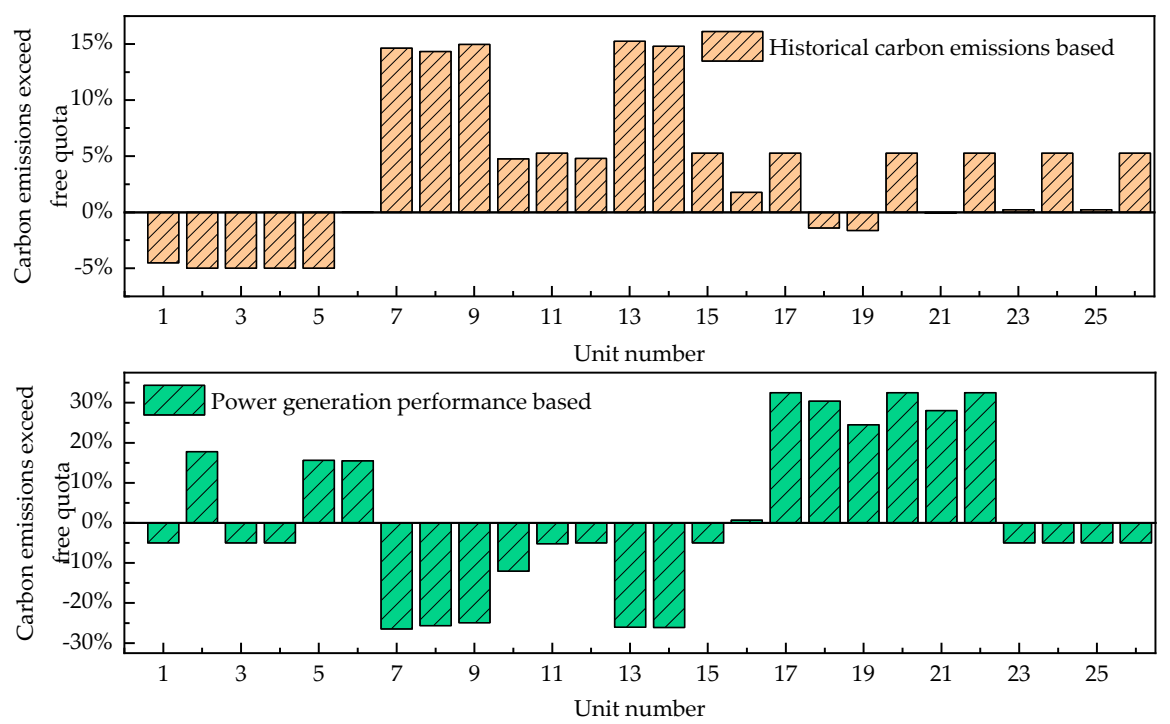

Figure 11. Comparison of carbon emissions of conventional units. 
Figure 12 displays the comparison of outputs of coal-fired units between two different carbon emission quota allocation methods. Due to the high carbon emission coefficient, the output of coal-fired units will be cut down to reduce their total carbon emission costs in the CET mechanism. The reduction of output is more obvious during the valley load periods. The output of coal-fired units will be further reduced (up to $59.62 \%$ ) if their quotas are allocated based on their power generation performance.

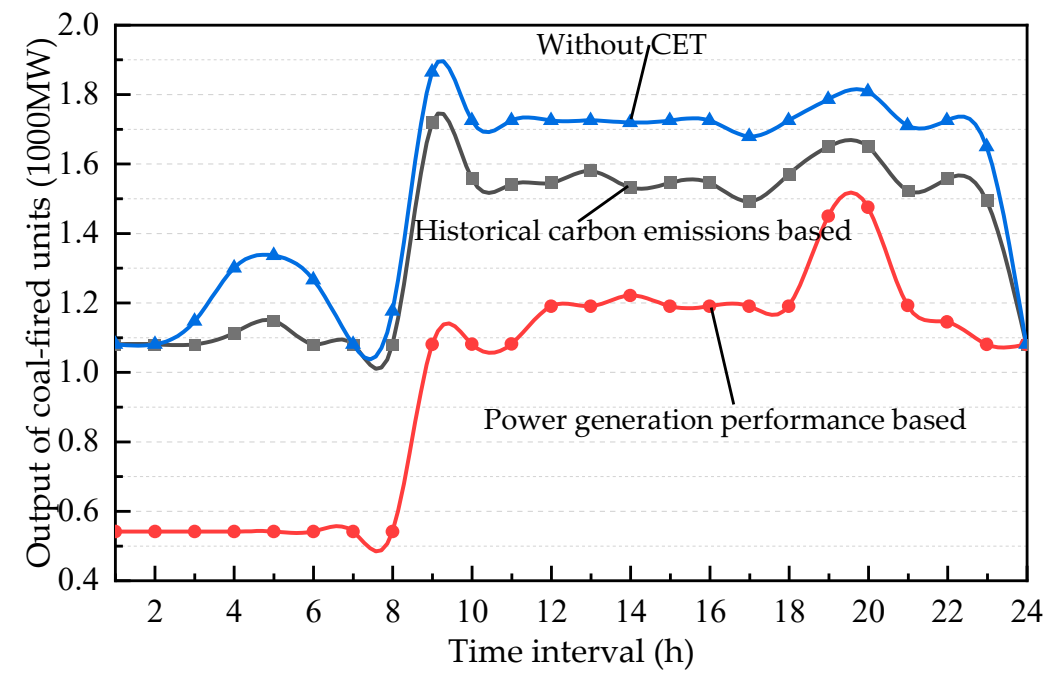

Figure 12. Comparison of outputs of coal-fired units.

Figure 13 shows the comparison of outputs of gas-fired units between two different carbon emission quota allocation methods. Although the marginal costs of gas-fired units are higher than those of coal-fired units, the carbon emission costs are lower due to their lower carbon emission coefficients. In order to meet the power balance constraints, the outputs of the gas-fired units will increase in the CET mechanism. The increase of gas-fired units' outputs can reach a maximum of $58.4 \%$ when the emission quotas are allocated based on power generation performance.

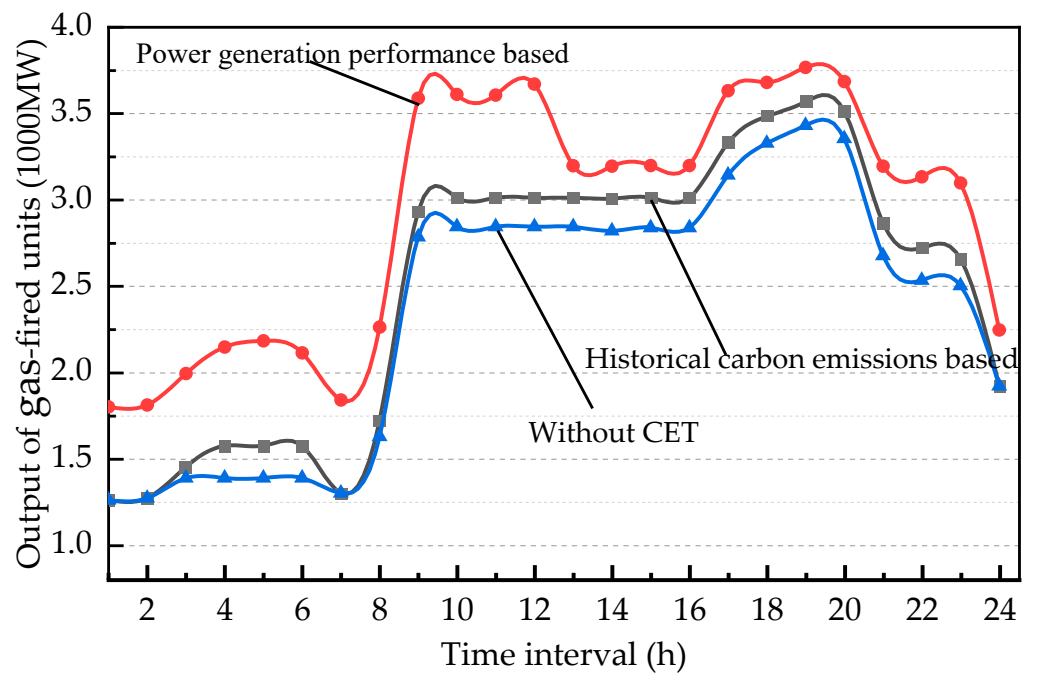

Figure 13. Comparison of outputs of gas-fired units.

Figure 14 demonstrates the comparison of outputs of renewable energy units between two different carbon emission quota allocation methods. Renewable units do not emit carbon during power generation. Due to the sufficient peak shaving capacity of the proposed system, the outputs of renewable units are not affected by the CET. 

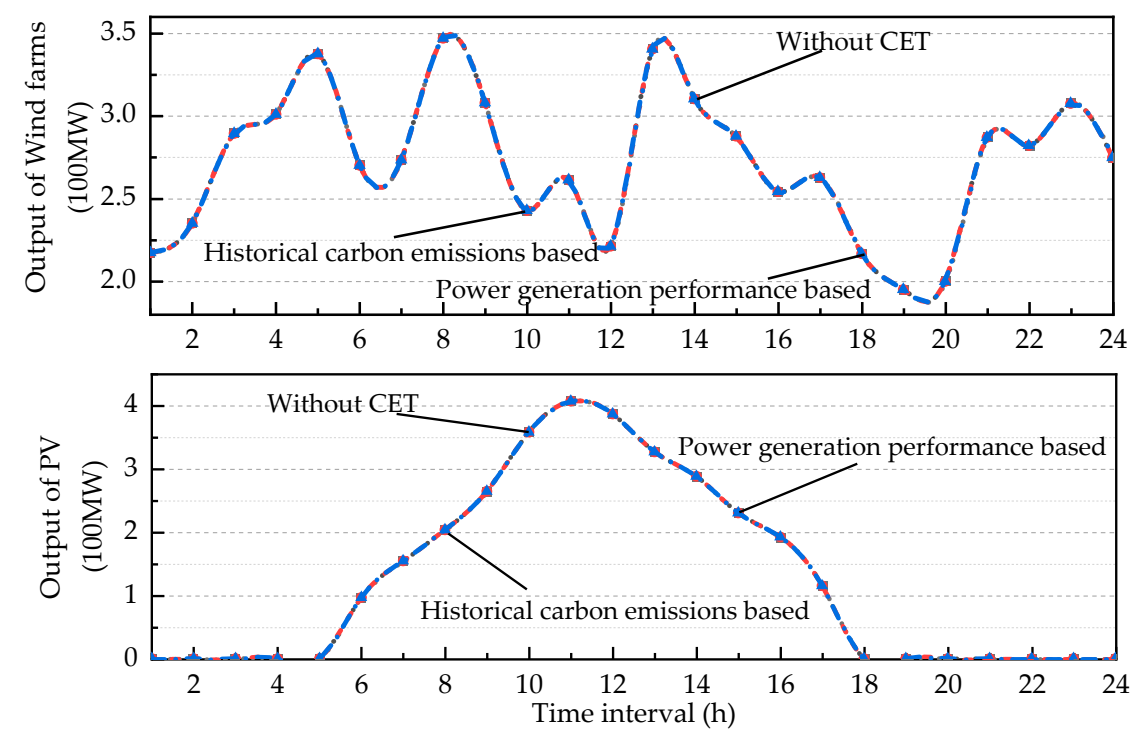

Figure 14. Comparison of outputs of renewable units.

Figure 15 presents the comparison of outputs of pumped storage power stations. The pumped storage units are all in charging mode during periods of valley load, and their outputs are exactly the same as the outputs without CET. When the emission quotas are allocated based on historical carbon emissions, the changes of outputs of conventional units are small during the peak load period, so the outputs of pumped storage units do not change significantly. However, the outputs of conventional units do change greatly due to the large difference in power generation performance with the power generation performance-based method. Therefore, the behavior of pumped storage units' output will also change significantly during the peak load period.

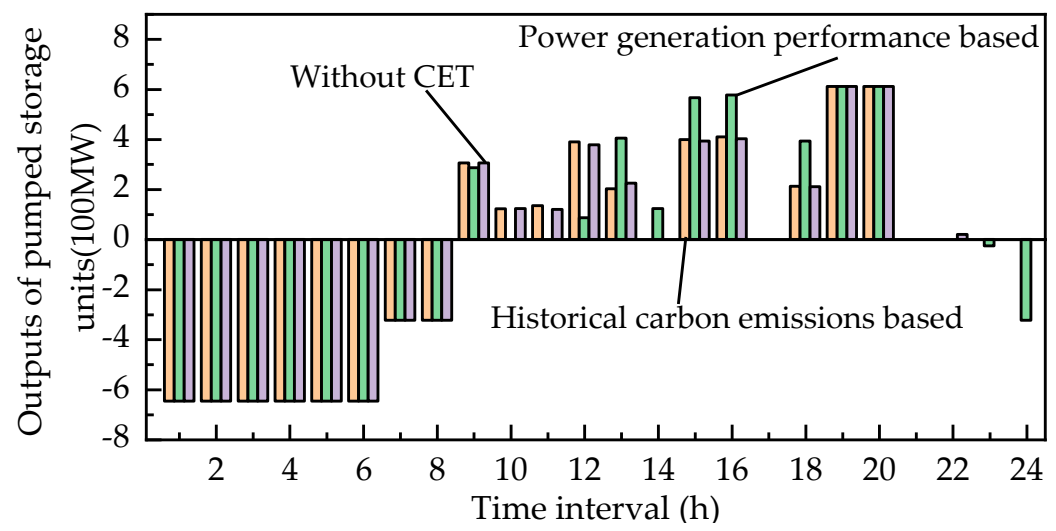

Figure 15. Comparison of outputs of pumped storage units.

Figure 16 presents the comparison of the LMP with different carbon emission quota allocation methods. When the emission quotas for conventional units are allocated by the power generation performance-based method, coal-fired units with lower generating costs obtain lower carbon quotas due to their lower power generation performance; hence, their quotation prices will significantly increase in the day-ahead market. The quotation prices of all conventional units in the market are at a relatively high level. Compared with the historical carbon emission-based method, the LMP of the electricity market has been increased, most significantly $(>62 \%)$ during peak load periods. 


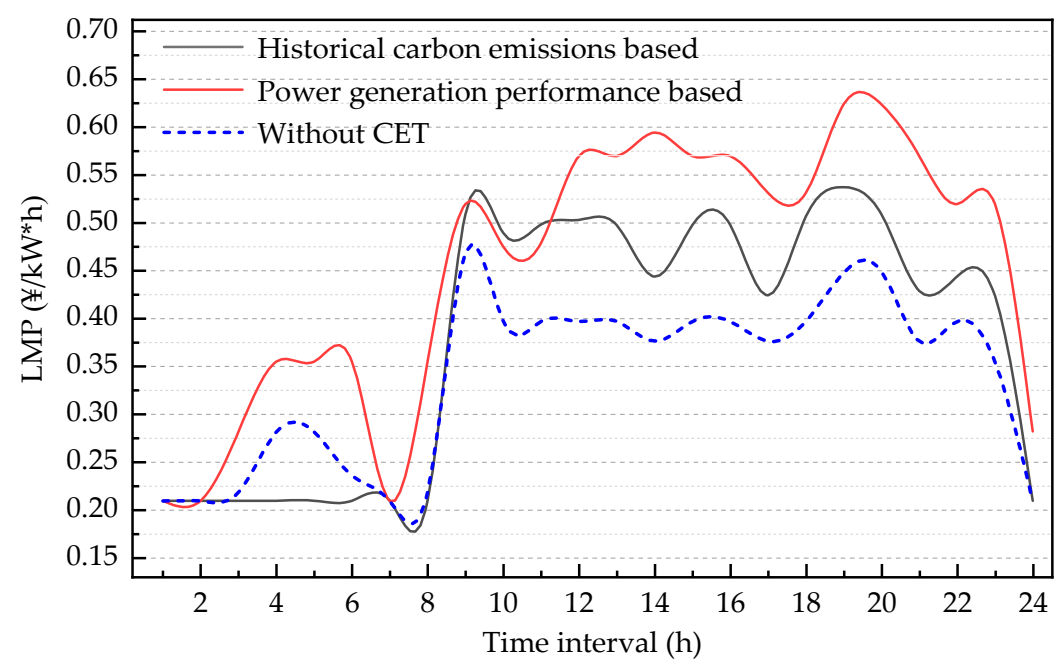

Figure 16. Comparison of locational marginal price (LMP) with different carbon emission quota allocation methods.

\section{Conclusions}

In this study, a two-stage model is formulated for the LMP calculation with CET. For the first stage of the model, a multi-objective optimization model to minimize both system operation costs and carbon emission costs is proposed and solved by the NNC method. For the second stage of the model, a tracing model with the goal of minimizing the total costs of the power system and satisfying the constraints generated by the Pareto optimal solution is built to calculate the LMP. Based on the data of a real-world 52-unit and 1560-bus system, the performance of the proposed methodology is studied in detail to analyze the impact of the CET.

According to the simulation, the outputs of coal-fired units are reduced, and the outputs of gas-fired units are increased. These results are even more pronounced in the allocation scheme based on the power generation performance-based method. The total operation costs of conventional units increase in the CET mechanism, and the quotation prices of conventional units increase in the day-ahead market, thereby raising the LMP sharply, especially during peak load periods. Furthermore, the LMP may rise up to $62 \%$ in the case of quotas allocated by the power generation performance-based method, which will significantly increase electricity costs and reduce social welfare. Therefore, this paper can provide a reference for the study of market clearing in consideration of the CET mechanism.

Author Contributions: Methodology, M.W., Z.L. and M.L.; Supervision, Q.C., T.Z., E.L. and W.L.; Writing-original draft, M.W., Z.L. and M.L.; Writing-review \& editing, M.W., Z.L. and M.L. All authors have read and agreed to the published version of the manuscript.

Funding: This research is funded by the Science and Technology Project of Guangdong Power Exchange Center Co. under Grant GDKJXM20172986.

Acknowledgments: The authors would like to thank the editor and reviewers for their sincere suggestions on improving the quality of this paper.

Conflicts of Interest: The authors declare no conflict of interest.

\section{References}

1. Liu, J.P.; Zhang, X.B.; Song, X.H. Regional carbon emission evolution mechanism and its prediction approach driven by carbon trading-A case study of Beijing. J. Clean. Prod. 2018, 172, 2793-2810.

2. Chen, X.; Lv, J.; McElroy, M.B.; Han, X.; Nielsen, C.P.; Wen, J. Power system capacity expansion under higher penetration of renewables considering flexibility constraints and low carbon policies. IEEE Trans. Power Syst. 2018, 33, 6240-6253. 
3. Lu, S.; Wu, Y.; Lou, S.; Yin, X. A model for optimizing spinning reserve requirement of power system under low-carbon economy. IEEE Trans. Sustain. Energy 2014, 5, 1048-1055.

4. Yuchi, Q.; Wang, N.; Li, S.; Yang, Z.; Jiang, B. A bi-objective reverse logistics network design under the emission trading scheme. IEEE Access 2019, 7, 105072-105085.

5. Huang, J.; Xue, Y.; Jiang, C.; Wen, F.; Xue, F.; Meng, K.; Dong, Z.Y. An experimental study on emission trading behaviors of generation companies. IEEE Trans. Power Syst. 2015, 30, 1076-1083.

6. Zhang, Z.; Zhu, K. Border carbon adjustments for exports of the United States and the European Union: Taking border-crossing frequency into account. Appl. Energy 2017, 201, 188-199.

7. Ding, G.; Deng, Y.; Lin, S. A study on the classification of China's provincial carbon emissions trading policy instruments: Taking Fujian province as an example. Energy Rep. 2019, 5, 1543-1550.

8. Lin, B.; Jia, Z. Is emission trading scheme an opportunity for renewable energy in China? A perspective of ETS revenue redistributions. Appl. Energy 2020, 263, 114605.

9. Wang, Y.; Qiu, J.; Tao, Y.; Zhao, J. Carbon-oriented operational planning in coupled electricity and emission trading markets. IEEE Trans. Power Syst. 2020. [CrossRef]

10. Zhou, X.; James, G.; Liebman, A.; Dong, Z.Y.; Ziser, C. Partial carbon permits allocation of potential emission trading scheme in Australian electricity market. IEEE Trans. Power Syst. 2010, 25, 543-553.

11. Yang, H.; Chung, C.Y.; Wong, K.P. Optimal fuel, power and load-based emissions trades for electric power supply chain equilibrium. IEEE Trans. Power Syst. 2012, 27, 1147-1157.

12. Li, X.R.; Yu, C.W.; Xu, Z.; Luo, F.J.; Dong, Z.Y.; Wong, K.P. A multimarket decision-making framework for genco considering emission trading scheme. IEEE Trans. Power Syst. 2013, 28, 4099-4108.

13. Kuo, M.T.; Lu, S.D.; Tsou, M.C. Considering carbon emissions in economic dispatch planning for isolated power systems: A case study of the taiwan power system. IEEE Trans. Ind. Appl. 2018, 54, 987-997.

14. D Olsen, D.J.; Dvorkin, Y.; Fernandez-Blanco, R.; Ortega-Vazquez, M.A. Optimal carbon taxes for emissions targets in the electricity sector. IEEE Trans. Power Syst. 2018, 33, 5892-5901.

15. Li, J.L.; Chen, Y.G.; Liu, S.j.; Wang, N.; Zou, P.; Chen, Q.X. Electricity market equilibrium analysis considering carbon emission cost. Power Syst. Technol. 2016, 40, 1558-1563.

16. Wang, T.; Jiang, C.W.; Li, L.; Zhao, Y. Security constrained unit commitment problem considering carbon emission allocation and demand side resources. Power Syst. Technol. 2016, 40, 3355-3361.

17. Fernández-Blanco, R.; Arroyo, J.M.; Alguacil, N. On the solution of revenue- and network-constrained day-ahead market clearing under marginal pricing-Part I: An exact bilevel programming approach. IEEE Trans. Power Syst. 2017, 32, 208-219.

18. Knueven, B.; Ostrowski, J.; Castillo, A.; Watson, J.P. A Computationally Efficient Algorithm for Computing Convex Hull Prices. Available online: http://www.optimization-online.org/DB_FILE/2019/09/7370.pdf (accessed on 9 January 2020).

19. Zhao, Z.; Wu, L. Impacts of high penetration wind generation and demand response on LMPs in day-ahead market. IEEE Trans. Smart Grid 2014, 5, 220-229.

20. Rosenthal, R.E. GAMS, A User's Guide; GAMS Development Corp.: Washington, DC, USA, 2014.

21. Li, Q.; Liu, M.; Liu, H. Piecewise normalized normal constraint method applied to minimization of voltage deviation and active power loss in an AC-DC hybrid power system. IEEE Trans. Power Syst. 2015, 30, 1243-1251.

22. Yu, S.; Wei, Y.M.; Guo, H.; Ding, L. Carbon emission coefficient measurement of the coal-to-power energy chain in China. Appl. Energy 2014, 114, 290-300.

23. Marler, R.T.; Arora, J.S. The weighted sum method for multi-objective optimization: New insights. Struct. Multidiscip. Optim. 2010, 41, 853-862.

24. Messac, A.; Ismail-Yahaya, A.; Mattson, C.A. The normalized normal constraint method for generating the Pareto frontier. Struct. Multidiscip. Optim. 2003, 25, 86-98.

(C) 2020 by the authors. Licensee MDPI, Basel, Switzerland. This article is an open access article distributed under the terms and conditions of the Creative Commons Attribution (CC BY) license (http://creativecommons.org/licenses/by/4.0/). 